\title{
Nash equilibria for non-binary choice rules
}

\author{
José C. R. Alcantud · Carlos Alós-Ferrer
}

\begin{abstract}
We prove the existence of equilibria in games with players who employ abstract (non-binary) choice rules. This framework goes beyond the standard, transitive model and encompasses games where players have nontransitive preferences (e.g., skew-symmetric bilinear preferences).
\end{abstract}

Keywords Non-cooperative games · Non-binary choice $\cdot$ SSB preferences

\section{JEL Classification $\quad \mathrm{C} 60 \cdot \mathrm{C} 72 \cdot \mathrm{D} 83$}

\section{Introduction}

The concept of Nash equilibrium and its refinements are based on assumptions of full rationality and common or mutual knowledge of the game on the part of the players (Aumann and Brandenburger 1995). The (empirically based and experimentally nourished) dissatisfaction of economists with such extreme assumptions has given rise to a large literature in which agents are assumed to display "bounded rationality" instead (see, e.g., Simon 1955). A large part of this literature is behavioral in nature, i.e., it conceives of agents as being focused on actions and not being necessarily able to predict the ultimate outcome. Technically, we can endow agents with a behavioral rule, which is merely a mapping

\section{J. C. R. Alcantud ( $\bowtie)$}

Facultad de Economía y Empresa, Universidad de Salamanca, Campus Miguel de Unamuno, 37007 Salamanca, Spain e-mail: jcr@usal.es

\section{Alós-Ferrer}

Department of Economics, University of Konstanz, Box D-150, 78457 Konstanz, Germany e-mail: Carlos.Alos-Ferrer@uni-konstanz.de 
from the current situation (e.g., the profile of actions taken by other players) to (a probability distribution over) actions. One such rule is, naturally, best reply, which in a sense puts classical game theory back into the picture, since Nash equilibria are the fixed points of this particular rule. Other rules that come to mind are imitation, better reply, and procedural rationality (in the sense of e.g., Osborne and Rubinstein 1998).

Here we investigate conditions for the existence of (generalized) Nash equilibria of games when (some of) the agents express their tastes on the prospective outcomes only through (not necessarily binary) choice rules. The use of the name "Nash" should not be taken as a reference to any optimization by the agents. We conceive of an equilibrium as a profile such that no single player wants to deviate; players' wishes, though, are directly modeled as choice rules, rather than being the result of optimization.

Bounded rationality is not only a concern of game theory but of all decision theory. Within this realm, a different branch of the literature has built upon the recognition that bounded rationality might still be captured by decision-theoretic models (cf. Rubinstein 1998, Chap. 1), leading to a re-examination of the common assumptions on the agents' perception of alternatives (preferences). For instance, the theory of skew-symmetric bilinear (SSB) preferences (see, e.g., Fishburn 1988) provides a framework for preferences which might fail one of the most basic rationality tests, that of transitivity (see also Shafer 1974).

An alternative approach to account for non-rational factors would be to move away from preferences, focusing instead on choices. Quoting Rubinstein (1998, pf. 7), "the most abstract model of choice refers to a decision maker who faces choices from sets of alternatives that are subsets of some grand set". Accordingly, agents are endowed with an abstract way to make choices which might not be supported by any underlying binary relation whatsoever (see, e.g., Nehring 1997). Technically, agents are now endowed with choice rules, ${ }^{1}$ which specify the acceptable choices given the range of available actions. These choice rules, rather than any underlying preferences, become the primitives of the model.

If we now consider agents interacting in a strategic situation, it is clear that there exists a strong analogy between the behavioral rules used in e.g., game-theoretic learning models and decision-theoretic choice rules. Formally, both are mappings from situations to actions. Conceptually, both are attempts to encompass bounded rationality within a general formal model of agents' behavior.

In the present work we make this analogy explicit and consider general games with players who employ abstract (non-binary) choice rules.

Beyond Nash's original contribution (Nash 1950, 1951), the existence of equilibria in games has been extensively justified on the basis of fixed point theorems. The first weakening was presented by Debreu (1952), who showed that the linearity assumption can be weakened to quasi-concavity. The transitivity assumption was relaxed by Kreweras (1961), Fishburn and Rosenthal (1986)

1 The term choice function is also used, but they are actually correspondences. In choosing the term choice rule, we follow Mas-Colell et al. (1995). 
(who considered non-transitive preferences of the SSB form), and Shafer and Sonnenschein (1975) (see also Border 1985, Corollary 19.4). Crawford (1990) analyzed preferences not satisfying the independence axiom. Dekel et al. (1991) considered certain non-expected utility preferences where violations of the reduction of compound lotteries assumption are allowed.

Of course, the concept of Nash equilibrum itself can be criticized, and the generality of these contributions does not mean that its existence is universally guaranteed. Crawford (1990) exhibits an example of non-existence when quasi-concavity fails. Another remarkable result concerning the non-existence of equilibrium is due to Ritzberger (1996), who considers the generalization of expected utility under rank-dependent probabilities.

The idea of a non-cooperative equilibrium as a situation where no player wishes to deviate is extremely compelling and has been taken beyond the framework of binary-relation maximization. Radner $(1980,1986)$ presented the idea of $\varepsilon$-equilibria, where players do not attempt to use every optimizing opportunity, but rather are satisfied with an approximately optimal strategy. Many other models have presented weakenings of full rationality without abandoning the idea of equilibrium. From the literature on learning in games, we single out Osborne and Rubinstein (1998). This work considers equilibria where players do not necessarily act on the basis of preference maximization, but rather follow certain, specific reasoning processes (procedural rationality).

The aim of this paper is quite specific. Relying on the recent literature on non-binary choice (Nehring 1996; Alcantud 2002), we provide a new equilibrium existence result. As pointed out later, such a result encompasses games where players have SSB preferences (which, in general, need neither be transitive nor satisfy the independence axiom), as well as Nash's theorem and the classical von Neumann-Morgenstern expected utility case.

\section{Choice rules and the equilibrium concept}

Consider an (abstract) set of players $I$. For each player $i \in I$, let $A_{i}$ denote her action set. Denote $A:=\prod_{i \in I} A_{i}$ and $A_{-i}:=\prod_{j \neq i} A_{j}$. We decompose $a=\left(a_{i}, a_{-i}\right)$ following the usual convention in game theory.

Denote by $\mathcal{P}^{*}\left(A_{i}\right)$ the set of all non-empty subsets of $A_{i}$. Let $\mathcal{D}_{i} \subseteq \mathcal{P}^{*}\left(A_{i}\right)$ be a collection of non-empty subsets of $A_{i}$ such that $A_{i} \in \mathcal{D}_{i}$. We call $\mathcal{D}_{i}$ the choice domain for player $i$.

A choice rule for player $i$ is a correspondence

$$
C_{i}: \mathcal{D}_{i} \times A_{-i} \longrightarrow A_{i}
$$

which depends on the actions of other players, and such that $C_{i}\left(D, a_{-i}\right) \subseteq D$ for each $D \in \mathcal{D}_{i}$ and $a_{-i} \in A_{-i}$.

For each $i \in I$ and $a_{-i} \in A_{-i}$, let

$$
C_{a_{-i}}: \mathcal{D}_{i} \longrightarrow A_{i}
$$


be the correspondence defined by $C_{a_{-i}}\left(D_{i}\right)=C_{i}\left(D_{i}, a_{-i}\right)$ on each $D_{i} \in \mathcal{D}_{i}$. Hence, we generalize the concept of a player's best reply to the other players' strategies (the vector $a_{-i}$ ), under the restriction that only a part of the action set (the subset $D_{i}$ ) is available, and according to the primitive choice correspondence $C_{i}$.

Abusing notation, we also denote $C_{i}\left(a_{-i}\right)=C_{a_{-i}}\left(A_{i}\right)$, the evaluation of the choice rule $C_{i}\left(\cdot, a_{-i}\right)$ on the full set $A_{i}$ for a given vector of strategies of the other players.

A (normal-form) game is a tuple $\left(I,\left\{A_{i}, C_{i}\right\}_{i \in I}\right)$ where $I$ is the set of players, the $A_{i}$ 's are their action sets, and the $C_{i}$ 's are the choice rules. A Nash equilibrium of this game is a profile $a^{*} \in A$ such that, for each $i \in I, a_{i}^{*} \in C_{i}\left(a_{-i}^{*}\right)$. In words, given the other players' strategies $\left(a_{-i}^{*}\right)$, player $i$ chooses $a_{i}^{*}$. Formally, a Nash equilibrium is a fixed point of the correspondence $C: A \longrightarrow A$ defined by $C(a)=\prod_{i \in I} C_{i}\left(a_{-i}\right)$ for each $a \in A$.

We make a number of restrictions. First, we consider a finite set of players, $I=\{1, \ldots, n\}$. Second, we restrict the sets $A_{i}$ to be simplexes over finite strategy sets. Namely, every $A_{i}$ is the simplex of mixed strategies on a set $S_{i}=\left\{s_{1}^{i}, \ldots, s_{m_{i}}^{i}\right\}$ for each $i$. Under this restriction, a choice rule models choices among mixtures of pure strategies. While this includes extensions of choices among pure strategies as in the von Neumann-Morgenstern case, a general choice rule on $A_{i}$ needs not be derived from a simpler rule on $S_{i}{ }^{2}$ Third, a topological structure will be assumed on each action set. We say that $C_{i}$ is upper semicontinuous if it has a closed graph in the product topology, that is, if $\left\{a^{k}\right\}_{k \in \mathbb{N}}$ is a sequence in $A$ converging to $a$ and $a_{i}^{k} \in C_{i}\left(a_{-i}^{k}\right)$ for each $k$, then $a_{i} \in C_{i}\left(a_{-i}\right)$.

\section{An existence result}

Following Nash (1950), Kakutani's fixed-point theorem is typically used to prove existence of a Nash equilibrium in the classical von Neumann-Morgenstern framework. The recourse to choice rules provides a richer framework allowing for non-binary preferences. Now the question arises as to which basic conditions on the choice rules ensure the existence of Nash equilibria.

The need to tackle non-binariness has long been recognized in economic theory. For instance, Gale and Mas-Colell (1975) provide a fixed-point Theorem which suffices to prove existence of Walrasian Equilibrium when the upper contour sets of consumers do not necessarily come from preference orderings. Border (1985, Chap. 19) explicitly refers to the lack of a non-binary background, stating that although it is "convenient to describe preferences in terms of the good reply correspondence rather than the preference relation [...] we lose some information by doing this." This position contrasts with Nehring (1997); this author argues that "the traditional assumption of binariness on preference

2 Recall that, under expected utility, the set of mixed-strategy Nash equilibria changes when the underlying utility defined on profiles of pure strategies is replaced by a different but (ordinally) equivalent utility function. Modeling choices among mixtures directly provides a conceptual way of avoiding this drawback without the recourse to cardinal utility. 
relations or choice functions is [...] analytically unhelpful and normatively unfounded [...]" on the basis e.g., of unresolvedness of preferences. "Dropping binariness," he says, "may lead to a deeper understanding of abstract choice theory."

Relying on Nehring (1996) and Alcantud (2002), we now present two sets of elementary conditions on choice rules and use them to provide new equilibrium existence theorems.

Our first set of conditions is based on Nehring (1996), who presents a model of non-binary choice which is appropriate for situations where agents are not compelled to choose mixtures of actions. The basic assumption is that agents are always able to make a choice when confronted with finitely many alternatives.

Condition 1 For each $i=1, \ldots, n$, the choice domain $\mathcal{D}_{i}$ includes all finite subsets of $A_{i}$. Further, for each $a_{-i} \in \prod_{j \neq i} A_{j}$, the following properties hold:

(a) If $S \subseteq A_{i}$ is finite, then $C_{a_{-i}}(S) \neq \varnothing$.

(b) For each $S, T \in \mathcal{D}_{i}: T \subseteq S$ implies $C_{a_{-i}}(S) \cap T \subseteq C_{a_{-i}}(T)$.

(c) For each $S \in \mathcal{D}_{i}$, if $a_{i} \in S$ is such that for each $T \subseteq S$ finite, $a_{i} \in T$ implies $a_{i} \in C_{a_{-i}}(T)$, then $a_{i} \in C_{a_{-i}}(S)$.

(d) For each $S \in \mathcal{D}_{i}$, if $\left\{a^{k}\right\}_{k \in \mathbb{N}}$ is a sequence in A converging to $a \in A$ such that $a_{i}^{k} \in C_{a_{-i}^{k}}\left(S \cup\left\{a_{i}^{k}\right\}\right)$ for each $k \in \mathbb{N}$, then $a_{i} \in C_{a_{-i}}\left(S \cup\left\{a_{i}\right\}\right) .^{3}$

(e) If $S \subseteq A_{i}$ is finite, then $M_{S}=\left\{a_{i} \in A_{i}: a_{i} \in C_{a_{-i}}\left(S \cup\left\{a_{i}\right\}\right)\right\}$ is convex. ${ }^{4}$

Let us briefly explain Condition 1 (for a more detailed discussion, see Nehring 1996). Part (a) presents the main idea (agents are always able to choose when confronted with a finite number of alternatives), which is 'non-emptiness' or A1 in Nehring (1996). Part (b) is sometimes called Chernoff's condition and is the rough analog of an "independence of irrelevant alternatives" assumption in this framework. It is called 'contraction consistency' or A2 in Nehring (1996). Part (c), which is 'finitariness' or A4 in Nehring (1996), implies that choice is based on finite sets (rather than pairs) of alternatives, in the sense that if an action is chosen whenever it is part of a finite subset of alternatives of a given set, then it must be chosen when the whole set is available. ${ }^{5}$

Part (d) is a technical requirement which parallels the continuity assumption A3 in Nehring (1996); see Lemma 3.1. Part (e) is the unavoidable convexity requirement.

Lemma 3.1 Suppose that $A_{i}$ and $C_{i}$ satisfy Condition 1(d). Then, for each $a_{-i}$, $M_{S}$ is closed for each finite $S \subseteq A_{i}$. Further, $C_{i}(\cdot)$ is upper semicontinuous.

Proof To see that $M_{S}$ is closed, apply Condition 1(d) with a fixed $a_{-i}^{k}=a_{-i}$. To see upper semicontinuity of $C_{i}$, apply Condition 1(d) with $S=A_{i}$.

3 Provided that $S \cup\left\{a_{i}^{k}\right\}, S \cup\left\{a_{i}\right\} \in \mathcal{D}_{i}$.

${ }^{4}$ We abuse notation by omitting the dependence of $M_{S}$ on $a_{-i}$.

5 In a sense, Condition 1(c) extends the range of $C_{a_{-i}}$ from finite to infinite sets. It is a weakening of 'binariness' or A5 in Nehring (1996). The latter, though, would produce a much more stringent framework; under non-emptiness and contraction consistency, it implies representability of $C$ through an acyclic binary relation (Nehring 1996, Remark 1). 
The following crucial property of the choice rules is a straightforward consequence of Lemma 3.1 plus the main theorem in Nehring (1996).

Lemma 3.2 Suppose $A_{i}$ and $C_{i}$ satisfy Conditions 1 (a)-(d). Then, for each $a_{-i} \in$ $\prod_{j \neq i} A_{j}$, the set $C_{i}\left(a_{-i}\right)$ is non-empty.

For the last lemma to hold true, compactness of each $A_{i}$ (rather than their simplicial form) would suffice.

Our second set of conditions is based on Alcantud (2002), who presents an alternative set of axioms which is more suitable for situations where agents naturally tend to use mixtures. For this approach, the natural set of alternatives is not taken to be a finite set as in Nehring (1996), but rather the infinite simplex of all mixtures of such a finite set. This is the inspiration for our second set of conditions. We denote by $\operatorname{co}(B)$ the convex hull of a set $B \subseteq A_{i}$ and $[x, y]=\operatorname{co}(\{x, y\})$.

Condition 2 For each $i=1, \ldots, n$, the choice domain $\mathcal{D}_{i}$ includes all convex hulls of finite subsets of $A_{i}$. Further, for each $a_{-i} \in \prod_{j \neq i} A_{j}, C_{a_{-i}}$ is such that the following properties hold for each $T=\left\{x_{1}, \ldots, x_{m}\right\} \subseteq A_{i}$ with $m \geqslant 2$.

(a) If $z \in \operatorname{co}(T)$, then there exists $k \in\{1, \ldots, m\}$ such that $z \in C_{a_{-i}}\left(\left[x_{k}, z\right]\right)$.

(b) For each $x \in A_{i}$, if $\left\{a^{k}\right\}_{k \in \mathbb{N}}$ is a sequence in $A$ converging to $a \in A$ such that $a_{i}^{k} \in C_{a_{-i}^{k}}\left(\left[x, a_{i}^{k}\right]\right)$ for each $k \in \mathbb{N}$, then $a_{i} \in C_{a_{-i}}\left(\left[x, a_{i}\right]\right)$.

(c) If $x_{j} \notin \operatorname{co}\left(T \backslash\left\{x_{j}\right\}\right)$ for each $j=1, \ldots, m$, then $C_{a_{-i}}(\operatorname{co}(T))=\bigcap_{j=1}^{m} M_{T}\left(x_{j}\right)$, where $M_{T}\left(x_{j}\right)=\left\{z \in \operatorname{co}(T) \mid z \in C_{a_{-i}}\left(\left[x_{j}, z\right]\right)\right\} .^{6}$

(d) For each $i \in I, M_{S_{i}}\left(s_{j}^{i}\right)$ is convex for each $j=1, \ldots, m_{i}$.

Let us now discuss Condition 2 (for more details, see Alcantud 2002). Part (a) says that, when considering a finite number of options, an agent can always declare a mixture $z$ eligible when compared with obtaining for sure some of the possible outcomes of $z$. Note, though, that the mixture $z$ needs not be preferred to all of the original options. That is, the rationality of the agent allows her to exclude at least one option from a finite number of alternatives.

Part (b) captures a certain continuity of the choice rule with respect to changes in the set of available alternatives. Indeed, together with part (c), it implies the following:

Lemma 3.3 Suppose that $A_{i}$ and $C_{i}$ agree with Condition 2(b). Then, for each $a_{-i}$, the corresponding set ${ }^{6} M_{T}\left(x_{k}\right)$ is closed for each $x_{k} \in T$. Further, if Condition 2(c) also holds, then $C_{i}(\cdot)$ is upper semicontinuous.

Proof To see that $M_{T}\left(x_{k}\right)$ is closed, apply Condition 2(b) with a fixed $a_{-i}^{k}=a_{-i}$. To see upper semicontinuity of $C_{i}$, notice that $C_{a_{-i}^{k}}\left(A_{i}\right)=\bigcap_{j=1}^{m_{i}} M_{S_{i}}\left(s_{j}^{i}\right)$ by Condition 2(c) (where the $M_{S_{i}}$ refer to $a_{-i}^{k}$ ), and apply Condition 2(b) to each $C_{a_{-i}^{k}}\left(\left[s_{j}^{i}, a_{i}^{k}\right]\right)$.

6 Again, we abuse notation by omitting the dependence of $M_{T}\left(x_{j}\right)$ on $a_{-i}$. 
Condition 2(c) states that, given the other players' strategies $a_{-i}$, player $i$ is willing to choose an alternative $z$ if and only if she is also willing to choose it when only mixtures of $z$ and an arbitrary pure strategy $s_{j}$ are allowed. As explained in Alcantud (2002), this property traces back choices to a "comparison with vertices" but does not imply binariness. ${ }^{7}$ We think that this is a weak rationality assumption, which is fulfilled in most normal-form game models.

Part (d) is a weak convexity requirement; notice that, due to the assumption on the form of the $A_{i}$ 's, it is only imposed on $M_{T}\left(x_{k}\right)$ where $T$ is the whole pure-strategy set and $x_{k}$ is a pure strategy.

Remark 3.4 In some particular cases, there is an interplay between parts (a) and (d). For instance, if one player has only two pure actions and maximizes complete and transitive preferences over mixtures, part (a) implies part (d). In general, though, these conditions are not related. To see this, consider first the two-player case where player 1 has three pure actions and player 2 has two pure actions. Hence $A_{1}$ is the simplex over three alternatives. Further, player 1 strictly prefers to randomize equiprobably [i.e., play $a_{1}=\left(\frac{1}{3}, \frac{1}{3}, \frac{1}{3}\right)$ ] independently of the actions $a_{2}$ of player 2. He is indifferent among all other actions. It is straightforward to check that Condition 2(a) is fulfilled. However, Condition 2(d) fails. To see this, denote by $e_{k}, k=1,2,3$, the three vertices of $A_{1}$. Then, $e_{2}, e_{3} \in M_{S_{1}}\left(e_{1}\right)$ but $z=\left(0, \frac{1}{2}, \frac{1}{2}\right) \notin M_{S_{1}}\left(e_{1}\right)$, because $C_{a_{2}}\left(\left[e_{1}, z\right]\right)=\left\{\left(\frac{1}{3}, \frac{1}{3}, \frac{1}{3}\right)\right\}$. Thus $M_{S_{1}}\left(e_{1}\right)$ is not convex. This shows that (a) does not imply (d) in general.

To see the converse, consider the two-player case where each player has exactly two pure actions, so $A_{1}=\{(p, 1-p) \mid 0 \leqslant p \leqslant 1\}$. Independently of the action $a_{2}$ of player 2 , player 1 maximizes preferences given by the utility function $u(p, 1-p)=-2 p$ for $p \leqslant \frac{1}{2}$ and $u(p, 1-p)=-2 p+2$ for $p>\frac{1}{2}$. Then, $M_{S_{1}}((0,1))=\{(0,1)\}$ and $M_{S_{1}}((1,0))=\left[\left(\frac{1}{2}, \frac{1}{2}\right),(1,0)\right]$, thus Condition 2(d) is fulfilled. But part (a) fails e.g., for $a_{1}=\left(\frac{1}{2}, \frac{1}{2}\right)$ and $T=S_{1}$.

Remark 3.5 It is worth discussing the particular case of players who maximize quasi-convex preferences (i.e., the lower contour sets are convex). As compared to the standard, quasi-concave case, this can be interpreted as aversion to probability mixing. As noted by Crawford (1990, Footnote 8), games with players having strictly quasi-convex preferences typically have only pure-strategy Nash equilibria or no Nash equilibrium at all.

First, we note that Condition 2(a) rules out strictly quasi-convex preferences, unless they are linear. For, if $\succcurlyeq$ is complete and transitive and there exist two indifferent elements $x \neq y$, part (a) implies that convex combinations of $x$ and $y$ are preferred to one of them, while strict quasi-convexity states that both $x$ and $y$ are strictly preferred to the convex combinations.

Further, under quasi-convex preferences Condition 2(a) implies part (d). To see this, fix $a_{-i}$ and $s=s_{j}^{i}$. Let $x, y \in M_{S_{i}}(s)$, that is, $x \in C_{a_{-i}}([x, s])$ and

7 Condition 2(c) is a strengthening of B1(b) in Alcantud (2002), which requires only the inclusion $\bigcap_{k=1}^{m} M_{T}\left(x_{k}\right) \subseteq C_{a_{-i}}(\operatorname{co}(T))$. If a choice correspondence is derived from a binary relation, the converse inclusion is fulfilled trivially. For such choice correspondences, requesting equality or only the inclusion mentioned above are equivalent. 
$y \in C_{a_{-i}}([y, s])$. Let $z=\lambda x+(1-\lambda) y$ for $\lambda \in[0,1]$. By Condition 2(a), we have w.l.o.g. that $z \in C_{a_{-i}}([z, x])$. In particular, $z \succcurlyeq x \succcurlyeq s$. By quasi-convexity $z \succcurlyeq t$ for all $t \in[z, s]$, implying that $z \in C_{a_{-i}}([z, s])$, i.e., $z \in M_{S_{i}}(s)$. This proves Condition 2(d).

Applying the results in Alcantud (2002), we obtain the following crucial property:

Lemma 3.6 Suppose $A_{i}$ and $C_{i}$ satisfy Conditions 2(a)-(c). Then, for each $a_{-i} \in$ $\prod_{j \neq i} A_{j}$, the set $C_{i}\left(a_{-i}\right)$ is non-empty.

Proof Conditions (a) and (c) above are B1(a) and B1(c) in Alcantud (2002). B2(a) in Alcantud (2002) holds when Lemma 3.3 applies. Then, Alcantud (2002, Theorem 1$)$ yields $C_{i}\left(a_{-i}\right)=C_{a_{-i}}\left(\operatorname{co}\left(s_{1}^{i}, \ldots, s_{m_{i}}^{i}\right)\right) \neq \varnothing$. Note that, by construction, the domain of each $C_{a_{-i}}$ satisfies the restriction imposed in that work (the convex hull of every finite set should be in the domain).

We are now ready to state the main existence result under non-binariness.

Theorem 3.7 Suppose that $\left(I,\left\{A_{i}, C_{i}\right\}_{i \in I}\right)$ satisfies either Condition 1 or 2 . Then the set of Nash equilibria is non-empty and compact.

Proof In order to apply Kakutani's fixed-point theorem to the correspondence $C: A \longrightarrow A$, we have to show that each $C_{i}$ is upper semicontinuous and also convex- and non-empty-valued. The latter condition holds by either Lemma 3.2 or 3.6. Upper semicontinuity follows from either Lemma 3.1 or 3.3.

We now prove that either of Conditions 1 or 2 imply that the $C_{i}$ 's are convexvalued. Under Condition 1, it follows from (d) and its consequences (namely, closedness of each $M_{S}$ ) plus (c) - see Remark 3 in Nehring (1996) - that

$$
C_{i}\left(a_{-i}\right)=\bigcap\left\{M_{S}: S \subseteq A_{i}, S \text { finite }\right\}
$$

and so the fact that $C_{i}\left(a_{-i}\right)$ is convex follows from (e). Under Condition 2, it follows from (c) and (d) that

$$
C_{a_{-i}}\left(A_{i}\right)=C_{a_{-i}}\left(\operatorname{co}\left(\left(s_{1}^{i}, \ldots, s_{m_{i}}^{i}\right)\right)\right)=\bigcap_{j=1, \ldots, m_{i}} M_{S_{i}}\left(s_{j}^{i}\right)
$$

is convex for each $i=1, \ldots, n$ and $a_{-i} \in \prod_{j \neq i} A_{j}$.

Finally, a standard result [e.g., Border 1985, 11.18(a)] assures that the set of fixed points of $C$ is closed in $A$, and hence compact (since $A$ is compact).

\section{Concluding remarks}

We have presented a general framework for normal-form games with players who follow arbitrary choice rules, which do not necessarily reflect optimizing behavior given some underlying preferences. The natural generalization of 
the concept of Nash equilibrium to this framework corresponds to a situation where no player wants to deviate given her choice rule. Even though such rule might actually be consistent with an underlying preference relation, the adopted behavioral rule might not correspond to pure optimization. For example, the choice rule might capture approximate optimization, as the one underlying the concept of $\varepsilon$-equilibrium (Radner 1980, 1986), or even complete departures from rationality, as e. g., rules based on imitation.

Since we consider choice rules as the result of some explicit non-binary choice procedure, we provide conditions based on the non-binary choice literature which lead to the existence of Nash equilibrium.

Straightforward application of Theorem 3.7 also allows us to derive many known results as Corollaries. For illustrative purposes, we make two of them explicit. We select these two examples because of their striking formal differences.

Consider first the "classical" case where players maximize a given preference relation. Under the assumptions of Debreu (1952) or Osborne and Rubinstein (1994, Proposition 20.3) (continuity of the preferences, quasi-concavity of each on the corresponding $A_{i}$, that is assumed to be a non-empty, compact and convex subset of a Euclidean space), Nash equilibria exist. It is not difficult to check that this case - which includes the original statement of Nash's equilibrium theorem - is encompassed by Theorem 3.7, in its version using Condition 1. This comes without surprise, since Nehring's (1996) result extended the Maximum Theorem of Bergstrom and Walker, which is the key for Kakutani's argument to apply. ${ }^{8}$

Second, recall that an SSB game is any game where players have a finite number of strategies (and hence every agent's action set is the simplex of mixed strategies) and players have SSB preferences. SSB preferences (e.g., Fishburn 1988) are characterized by a SSB function $\phi$ in the following way: an alternative $x$ is (weakly) SSB-better than another one, $y$, if and only if $\phi(x, y) \geqslant 0$. Such preferences [see Shafer (1974) for a straightforward representation theorem] need neither be transitive nor satisfy the independence axiom, although the classical von Neumann-Morgenstern framework is encompassed as a particular case. ${ }^{9}$ Kreweras (1961) (see also Fishburn and Rosenthal 1986) already obtained existence of Nash equilibria in SSB games. It is straightforward to check that this existence result is also a corollary to Theorem 3.7 (just proceed with the choice rules given by $C_{a_{-i}}\left(D_{i}\right)=\left\{x_{i} \in D_{i}: \phi_{i}\left(\left(x_{i}, a_{-i}\right),\left(y_{i}, a_{-i}\right)\right) \geqslant 0\right.$ for each $\left.y_{i} \in D_{i}\right\}$, for each $D_{i} \subseteq A_{i}$ and $a_{-i}$ ).

8 In fact, Condition 2 is fulfilled too, except part (c). This is also satisfied if not only $\left\{a_{i} \in A_{i}\right.$ : $\left.\left(a_{i}, a_{-i}^{*}\right) \succcurlyeq_{i} a^{*}\right\}$, but also $\left\{a_{i} \in A_{i}: a^{*} \succcurlyeq_{i}\left(a_{i}, a_{-i}^{*}\right)\right\}$, are convex for each $a^{*} \in A$. The latter condition holds in the classical von Neumann-Morgenstern expected utility case. Further, the comment on the definition of condition B1 in Alcantud (2002) puts forward a number of preference classes that satisfy the additional requirement.

9 Renewed interest on the SSB model has arisen recently in the framework of dynamic models for selection of preferences. See Cubitt and Sugden (1998) and Alós-Ferrer and Nermuth (2003). 
Acknowledgments We thank Manfred Nermuth, Klaus Ritzberger, an anonymous referee, an associate editor, and William Thomson, the Editor of the Journal, for helpful comments. Part of this work was done while the first author was visiting the University of Vienna under the Spain-Austria Acciones Integradas respective projects HU02-4 and 18/2003. This author has benefited from financial support from FEDER and the Ministerio de Ciencia y Tecnología under the Research Project SEJ2005-03041/ECON, the Junta de Castilla y León under the Research Project SA098A05, and Ministerio de Educación y Ciencia (European Science Foundation under Acción Especial SEC2002-10181-E). The second author acknowledges financial support from the European Science Foundation and the Austrian Science Fund (FWF), under Project P15281.

\section{References}

Alcantud JCR (2002) Non-binary choice in a non-deterministic model. Econ Lett 77(1):117-23

Alós-Ferrer C, Nermuth M (2003) A comment on 'The Selection of Preferences through Imitation'. Econ Bull 3:1-9

Aumann R, Brandenburger A (1995) Epistemic conditions for Nash equilibrium. Econometrica 63(5):1161-1180

Border KC (1985) Fixed point theorems, with applications to economics and game theory. Cambridge University Press, Cambridge, MA

Crawford VP (1990) Equilibrium without independence. J Econ Theory 50:127-154

Cubitt RP, Sugden R (1998) The selection of preferences through imitation. Rev Econ Stud 65: 761-771

Debreu G (1952) A social equilibrium existence theorem. Proc Natl Acad Sci 38:886-893

Dekel E, Safra Z, Segal U (1991) Existence and dynamic consistency of Nash equilibrium with non-expected utility preferences. J Econ Theory 55:229-246

Fishburn PC (1988) Nonlinear preference and utility theory. Wheatsheaf Books, New York

Fishburn PC, Rosenthal RW (1986) Noncooperative games and nontransitive preferences. Math Soc Sci 12:1-7

Gale D, Mas-Colell A (1975) An equilibrium existence theorem for a general model without ordered preferences. J Math Econ 2:9-15

Kreweras G (1961) Sur une possibilite de rationaliser les intransitivites. In: La Decision. Colloques Internationaux du Centre National de la Recherche Scientifique, Editions du Centre National de la Recherche Scientifique. Paris, pp $27-32$

Mas-Colell A, Whinston MD, Green JD (1995) Microeconomic theory. Oxford University Press, Oxford

Nash JF (1950) Equilibrium points in N-person games. Proc Natl Acad Sci 36:48-49

Nash JF (1951) Non-cooperative games. Ann Math 54:286-295

Nehring K (1996) Maximal elements of non-binary choice functions on compact sets. Econ Lett 50:337-340

Nehring K (1997) Rational choice without binariness. Soc Choice Welf 14:403-425

Osborne MJ, Rubinstein A (1994) A course in game theory. MIT Press, Cambridge, MA

Osborne MJ, Rubinstein A (1998) Games with procedurally rational players. Am Econ Rev 88: 834-847

Radner R (1980) Collusive behavior in noncooperative epsilon-equilibra of oligopolies with long but finite lives. J Econ Theory 22:136-154

Radner R (1986) Can bounded rationality resolve the prisoner's Dilemma? In: Hildenbrand W, Mas-Colell A (eds) Contributions to mathematical economics. Essays in honor of G. Debreu. North-Holland, Amsterdam, pp 387-399

Ritzberger K (1996) On games under expected utility with rank dependent probabilities. Theory Decis 40:1-27

Rubinstein A (1998) Modeling bounded rationality. MIT Press, Cambridge, MA

Shafer WJ (1974) The nontransitive consumer. Econometrica 42:913-919

Shafer WJ, Sonnenschein H (1975) Equilibrium in abstract economies without ordered preferences. J Math Econ 2:345-348

Simon HA (1955) A behavioral model of rational choice. Q J Econ 69:99-118 